\title{
A central role for TOR signalling in a yeast model for juvenile CLN3 disease
}

\author{
Michael E. Bond ${ }^{1}$, Rachel Brown ${ }^{1}$, Charalampos Rallis ${ }^{3,4}$, Jürg Bähler ${ }^{3,4}$ and Sara E. Mole , $^{1,2,3, *}$ \\ ${ }^{1}$ MRC Laboratory for Molecular Cell Biology, University College London, London WC1E 6BT, UK. \\ ${ }^{2}$ UCL Institute of Child Health, 30 Guilford Street, London WC1N 1EH, UK. \\ ${ }^{3}$ Department of Genetics, Evolution and Environment, University College London, London WC1E 6BT, UK. \\ ${ }^{4}$ Institute of Healthy Ageing, University College London, London WC1E 6BT, UK. \\ * Corresponding Author: Sara E. Mole, MRC Laboratory for Molecular Cell Biology, University College London; London WC1E 6BT, UK; \\ Tel: +44 207679 7257; Fax: +44 207679 7805; E-mail: s.mole@ucl.ac.uk
}

\begin{abstract}
Yeasts provide an excellent genetically tractable eukaryotic system for investigating the function of genes in their biological context, and are especially relevant for those conserved genes that cause disease. We study the role of btn1, the orthologue of a human gene that underlies an early onset neurodegenerative disease (juvenile CLN3 disease, neuronal ceroid lipofuscinosis (NCLs) or Batten disease) in the fission yeast Schizosaccharomyces pombe. A global screen for genetic interactions with btn1 highlighted a conserved key signalling hub in which multiple components functionally relate to this conserved disease gene. This signalling hub includes two major mitogen-activated protein kinase (MAPK) cascades, and centers on the Tor kinase complexes TORC1 and TORC2. We confirmed that yeast cells modelling CLN3 disease exhibit features consistent with dysfunction in the TORC pathways, and showed that modulating TORC function leads to a comprehensive rescue of defects in this yeast disease model. The same pathways may be novel targets in the development of therapies for the NCLs and related diseases.
\end{abstract}

doi: $10.15698 /$ mic2015.12.241

Received originally: 06.08.2015;

in revised form: 12.10.2015,

Accepted 14.10.2015,

Published 11.11.2015.

Keywords: Batten disease, NCL, CLN3, btn1, Tor, TORC, S. pombe, yeast.
Abbreviations:
CWI - cell wall integrity,
NCLs - neuronal ceroid lipofuscinosis, SAPK - stress-associated protein kinase, $S G A$ - synthetic genetic array, $W T$ - wild-type.

\section{INTRODUCTION}

Yeasts have long been used as model systems to shed light on basic eukaryotic cell biology, and can provide a rapid and comprehensive route of investigation for genes of unknown function. This is particularly relevant for those genes that are conserved across diverse eukaryotic species and which can be presumed to play a fundamental biological role. A significant health challenge facing current research and drug development is the increasing incidence of age-related neurodegenerative disorders. Elucidating the mechanisms that underlie neurodegeneration is complex, particularly so in common age-related dementias that may have numerous contributing factors. There are, however, monogenic inherited neurodegenerative diseases that present a simpler alternative for investigation and real opportunities to determine these basic cellular changes, and some of the underlying genes are conserved even in yeasts. The genetic tractability of yeasts offers particular advantages to the challenges of understanding disease mechanisms in a relevant biological context and this knowledge can inform therapeutic development for such conserved genetic diseases.

The neuronal ceroid lipofuscinoses (NCLs) are such a group of monogenic neurodegenerative disorders that generally affect children [1]. The most common of these is juvenile CLN3 disease [2]. This disease is characterised by progressive neuronal atrophy that causes visual failure, seizures and a progressive decline in cognitive and motor function. This disease is accompanied by cellular features characteristic of many neurodegenerative conditions $[3,4]$ that include the dysfunction of core cellular processes, such as reduced lysosomal and autophagic clearance $[5,6]$ and mitochondrial abnormalities [7]. Moreover, CLN3 disease also leads to specific cellular pathologies characteristic of more common dementias. These include the accumulation of lipofuscin [1], as observed in aged neurons [4], aberrant amyloid- $\beta$ processing [5], a feature of Alzheimer's disease [8] and $\alpha$-synuclein accumulation [9], a feature of Parkinson's disease [10].

Juvenile CLN3 disease is caused by mutations in a single gene (CLN3) [2], whose function is unknown but which is 


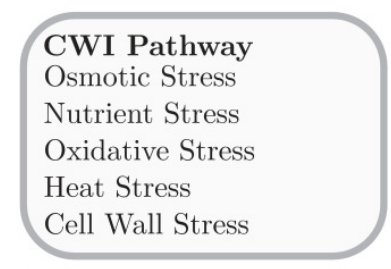

\section{SAPK Pathway}

Osmotic Stress

Nutrient Stress

Oxidative Stress

Heat Stress
Cell Wall Stress

DNA Replication Stress



CELL WALL

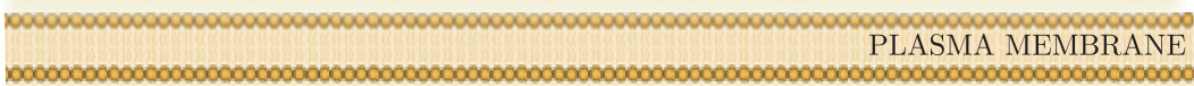

CYTOPLASM

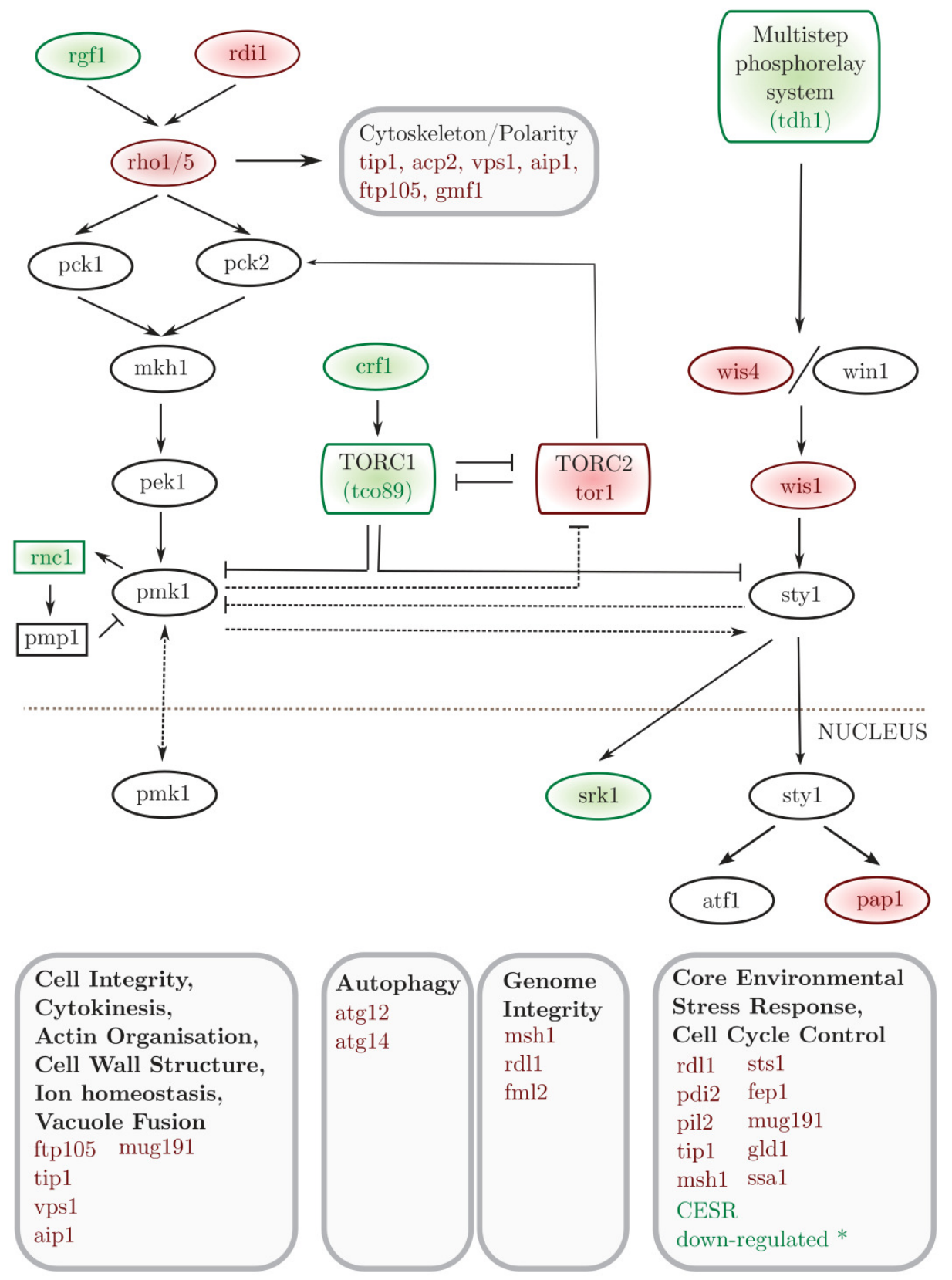

FIGURE 1: Synthetic genetic array (SGA) analysis of the genetic interactions of $b t n 1$. A schematic of the genes involved in stress responses that genetically interact with $b t n 1$. Genes in red were identified as negative interactors and those in green as positive. Grey genes are involved in these pathways but were not found to interact with btn1. * CESR refers to 'core environmental stress response' genes, a group of genes that respond to most environmental stressors as described by Chen et al. (2003) [76]. 
highly conserved across eukaryotic species [11]. As a consequence, this disease is relatively straightforward to reproduce in experimental systems and, given its similarity to more common dementias, such experimental models are ideal paradigms to study the basic cellular changes that occur in neurodegenerative diseases. The fission yeast Schizosaccharomyces pombe contains a single orthologue of CLN3 (btn1) [11]. Work in this yeast has revealed a role for btn1 in many cellular processes. Like patient cells, yeast lacking btn 1 (btn $1 \Delta)$ have enlarged and less acidic vacuoles [12] and further work has supported roles for btn 1 in vacuolar homeostasis [13]. The ability of btn 1 modeling the most common CLN3 mutation (a $1 \mathrm{~Kb}$ deletion) to rescue these vacuolar defects found in the $b \operatorname{tn} 1 \Delta$ null strain has also revealed that this mutant allele retains some function, making juvenile CLN3 disease a mutation-specific disease rather than the consequence of a complete loss of function as originally assumed [14].

A host of further morphological defects exhibited in the $b t n 1 \Delta$ strain support additional roles for btn 1 in cytokinesis [12] and the organisation of cell polarity [15]. Furthermore, btn $1 \Delta$ cells also display cell wall defects [16]. Importantly, work in this model has expanded our understanding of the disease by revealing that $b t n 1$ is involved in two independent pathways; one $\mathrm{pH}$-dependent and one $\mathrm{pH}$-independent, thus providing the first suggestion that Batten disease is more than a $\mathrm{pH}$-related lysosome disorder [16]. Indeed, a comprehensive metabolomics approach has revealed that btn 1 is required for the regulation of glycolysis and amino acid homeostasis [17]. The involvement of btn1 in numerous, apparently disparate, pathways may be a result of alterations at the Golgi apparatus, as the number, morphology, and location of thus organelle are affected by its deletion [13]. Lastly, this model has been used successfully to model disease mutations in Btn1p, an effort that has provided valuable insight into their consequences on protein trafficking and function. It was found that equivalent CLN3 disease mutations in btn1 affect the yeast phenotype in a way that can accurately predict the severity of disease, further establishing yeast as an accurate disease model despite its simplicity. Importantly, the observations reported in the fission yeast model have consistently been confirmed in mammalian systems [5, 18, 19]. Unfortunately however, despite these insights, the molecular processes that underlie cell death in this disease are poorly understood, the function of CLN3 is unknown, and there remains a significant need for protective therapeutic targets.

A particular advantage of yeast model systems is the availability of genome-wide techniques. Synthetic genetic arrays (SGAs) have proved a particularly powerful means of exploring genetic interactions in yeast species [20]. This approach highlights genes involved in pathways parallel to, or converging with, the query gene. This provides information about functional relationships among genes, as well as processes that suppress the defects associated with a particular mutation. As they are hypothesis-free, SGAs are particularly valuable in the investigation of complex biological problems and those where gene function is unclear. These advantages are particularly relevant for neu- rodegeneration in general, due to the complexity of the problem, and for juvenile CLN3 disease in particular, due to the lack of a clear gene function. SGAs have previously been employed in budding yeast to investigate mutant huntingtin and $\alpha$-synuclein toxicity [21]. Such an approach can place the gene under investigation within its biological context and thereby uncover much-needed protective pathways for neurodegenerative disease.

We applied SGA analysis to identify pathways that are altered as a consequence of loss of function of btn 1 in an effort to better understand the molecular consequences of CLN3 disease, and to provide new candidate target pathways and processes for therapeutic development. A third of the genetic interactions that were identified centered on a set of conserved and connected signalling pathways. Manipulation of these pathways leads to a complete rescue of the pleiotropic array of btn $1 \Delta$ phenotypes. This approach represents the most successful rescue of cellular dysfunction in any model for juvenile CLN3 disease to date.

\section{RESULTS}

Genome-wide analysis of genetic interactions with btn1 reveals a central role for TOR kinases

We applied an SGA approach as an unbiased, genome-wide strategy to probe the interactions of a conserved neurodegenerative disease gene (btn1). We identified 331 positive interactors and 131 negative interactors of btn 1 (Table $\mathrm{S} 1 \mathrm{~A}$ and $\mathrm{B}$ ). A large number of negative interactions ( $\mathrm{n}=$ $39 ; 29.7 \%$ ) mapped to a set of highly interconnected signalling processes, which center on the Tor kinase complexes TORC1 and TORC2 (Fig. 1). Core components of both complexes display genetic interactions with btn1; tco89, encoding a component of TORC1, was found to interact positively with btn1, while tor1, encoding the Tor kinase of TORC2, was found to interact negatively with $b t n 1$. Such a pattern suggests that TORC2 signalling is beneficial to the fitness of btn1 $1 \Delta$ cells, whereas TORC1 signalling is detrimental, an observation that is in keeping with the idea that the two complexes play opposing roles and undergo mutual repression [22-24]. btn1 was also found to negatively interact with genes encoding components of the connected cell wall integrity (CWI) and stress-associated protein kinase (SAPK) MAP kinase cascades, and a number of downstream stress regulated genes (Table S1A and B).

\section{btn1 $\Delta$ cells display features consistent with dysfunctional Tor signalling}

The interaction of btn 1 with core TORC components, and the link to surrounding signalling processes, provides compelling evidence for the importance of Tor signalling in cells lacking btn1. To validate this observation, we investigated btn1 $1 \Delta$ cells for features consistent with Tor dysfunction. Repression of TORC1 activity is required to mount a correct response to nitrogen limitation [24]. As loss of TORC1 activity was beneficial in cells lacking btn1, we hypothesised that these cells may display features consistent with dysregulation of TORC1 repression, and respond poorly to nitrogen limitation. To test this idea, we grew wild-type 
and btn $1 \Delta$ cells in minimal media, and minimal media lacking a nitrogen source, over $72 \mathrm{~h}$. Viability was monitored at $24 \mathrm{~h}$ intervals using propidium iodide to label dead cells and calcofluor white to label the total cell population (Fig. 2A). Cells lacking btn 1 displayed a consistently lower viability in media lacking nitrogen, falling to $81.5 \pm 2.2 \%$ after 24 h compared to $98.7 \pm 0.27 \%$ in minimal media containing nitrogen $(P<0.0015$, unpaired $t$ test $)$, and remaining lower throughout the time course. Wild-type cells displayed no change in viability when cultured in media lacking nitrogen.

TORC1 repression and TORC2 signalling is also required to mount a response to glucose limitation $[25,26]$. Cells that are defective in TORC2 function do not respond appropriately to glucose limitation by reducing their cell length [26]. This is a relatively uncommon phenotype, with only tor1 mutants and mutants in the $\mathrm{Ca}^{2+} /$ calmodulindependent-like gene ssp1 known to display this phenotype $[26,27]$. To test the ability of $b t n 1 \Delta$ cells to adapt to conditions of limited glucose, we assessed both cell growth and the morphological response of these cells when grown with an alternative carbon source (glycerol). Cells lacking btn1 displayed a clear growth defect under these conditions (Fig. 2B). Wild-type and btn $1 \Delta$ cells grown for $6 \mathrm{~h}$ in media containing either glucose or glycerol as a carbon source were stained with calcofluor white to visualise the cell wall. Cell elongation was determined using a measure of cell circularity and given a score of between 0 and 1 , where 0 represents a perfectly round cell (Fig. 2C and D). Wild-type cells displayed a significant reduction in cell elongation upon growth in glycerol $(0.25 \pm 0.01$ to $0.16 \pm$ $0.01, P \leq 0.01)$. Cells lacking btn1, however, displayed no significant change $(0.32 \pm 0.02$ to $0.31 \pm 0.02)$, consistent with a defect in their response to glucose limitation that is most likely linked to Tor1 function.

Lastly, TORC2 and the connected CWI pathway are also required for resistance to high temperature [26]. This temperature sensitivity of TORC2 and CWI pathway components is osmoremedial, i.e. it can be rescued by hypertonic growth media [28]. We confirmed the temperature sensitivity of btn1 $1 \Delta$ cells, and that it can be rescued with $2 \mathrm{M}$
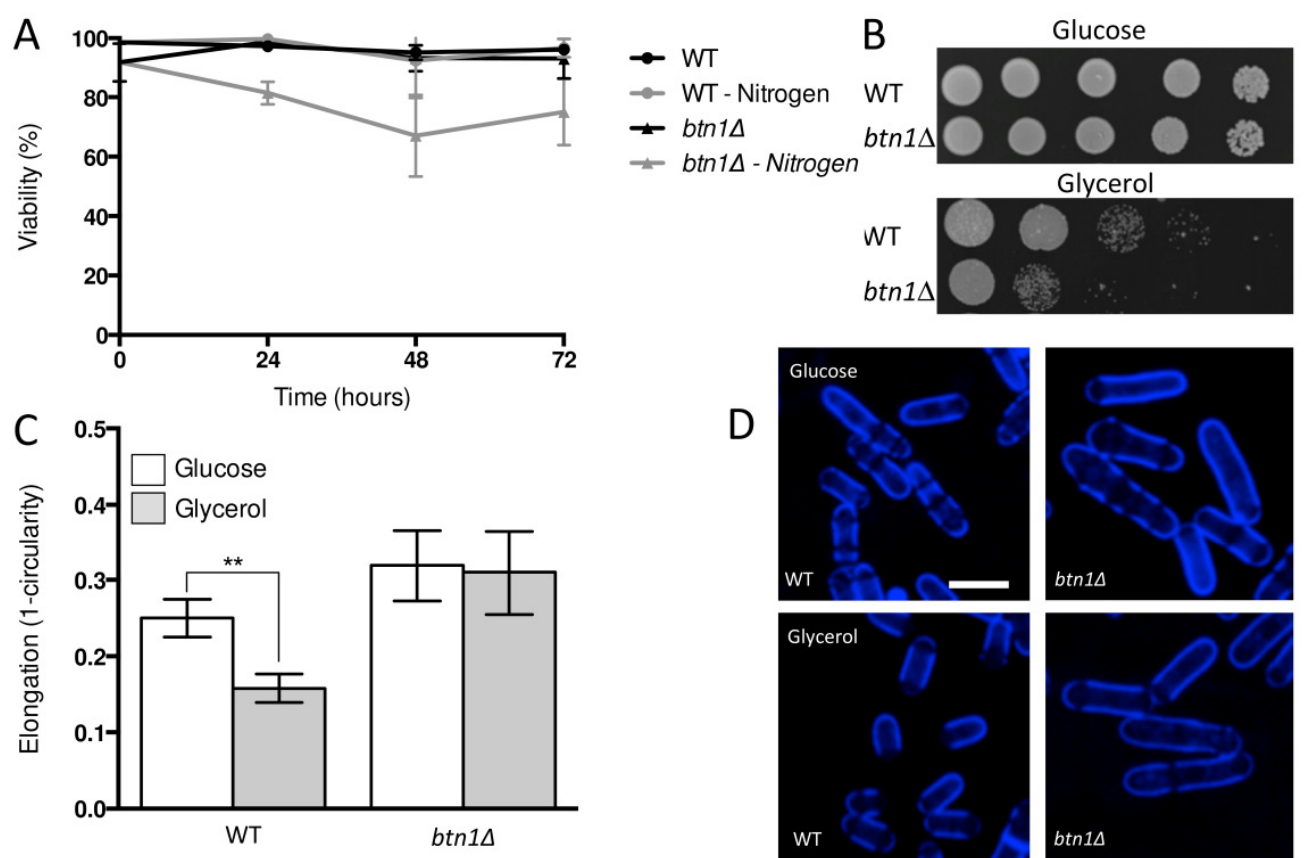

Glycerol
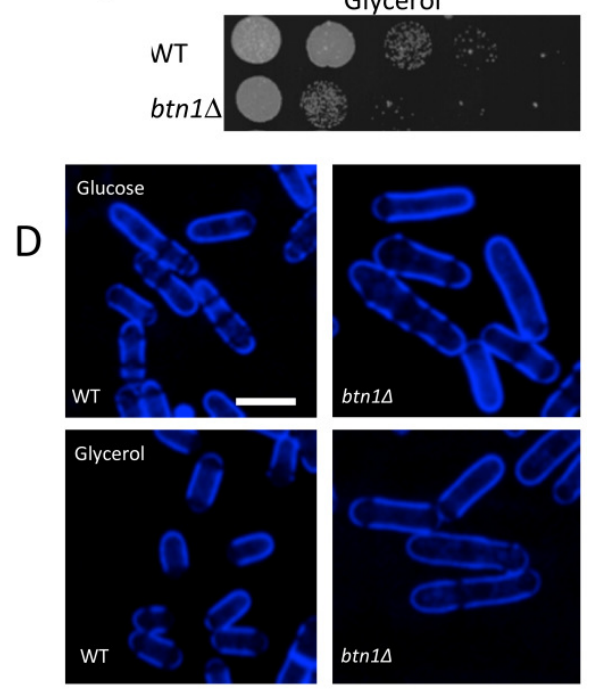

E
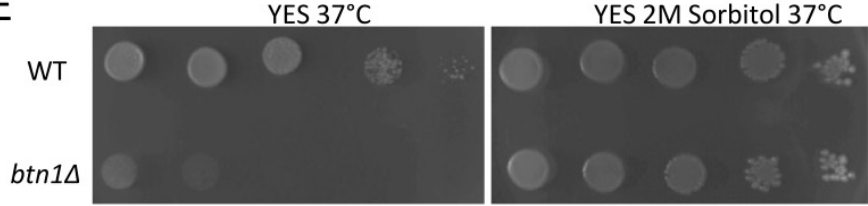

FIGURE 2: btn1s cells display features consistent with dysfunction in TOR signalling processes. (A) Cell viability upon nitrogen limitation was determined over periods of up to 72 hours in wild-type (WT) and btn1s cells, using the cell impermeable nucleotide stain propidium iodide to stain dead cells and calcofluor white to stain the total cell population. Cells were cultured in either MM or $\mathrm{MM}$ lacking a nitrogen source $\left(\mathrm{NH}_{4} \mathrm{Cl}\right)$. 500 cells were scored for viability per data set, and data shown is a mean $( \pm$ SEM) of 3 independent experiments. (B) WT cells and btn $1 \Delta$ cells were serially diluted from a log-phase culture $\left(1 \times 10^{6}\right.$ cells $/ \mathrm{ml}$ ), and spotted onto plates containing either glucose or glycerol as a carbon source. Plates were then incubated at $30^{\circ} \mathrm{C}$ for 6 - 7 days to determine growth on fermentative and non-fermentative car-

bon sources. Images are representative of three independent experiments. (C) The morphological response of WT and btn1 $\Delta$ cells to growth on glycerol was analysed following 6 hours in culture using a measure of cell elongation, on a scale of 0 to 1 , where 0 represents a perfectly round cell $\left(1-\left(\frac{4 \pi \text { area }^{2}}{\text { perimeter }^{2}}\right)\right.$. Data shown is a mean $( \pm S E M)$ of 5 independent experiments. Statistical significance between each condition was determined using a one-way ANOVA with a Tukey's multiple comparison post-test $(* *=P<0.01)$. (D) Representative images of experiments as performed in (C) are shown. Scale bar represents $10 \mu \mathrm{m}$. (E) WT and btn $1 \Delta$ cells were also serially diluted from a log-phase culture $\left(1 \times 10^{6} \mathrm{cells} / \mathrm{ml}\right)$ and spotted onto YES plates and YES plates containing $2 \mathrm{M}$ sorbitol. Plates were then incubated at $37^{\circ} \mathrm{C}$ for $3-4$ days to determine the growth at high temperature and the influence of osmotic stabilisation. Images are representative of three independent experiments. 
sorbitol (Fig. 2E), consistent with previous observations [16].

These data together indicate that cells lacking btn1 display many features consistent with defects in Tor signalling and function. Further, ectopic expression of btn 1 was able to rescue all these aspects of the mutant phenotype (Fig. S1A - D). Combined with the SGA results, these results suggest that Tor signalling is critical to the defects observed in
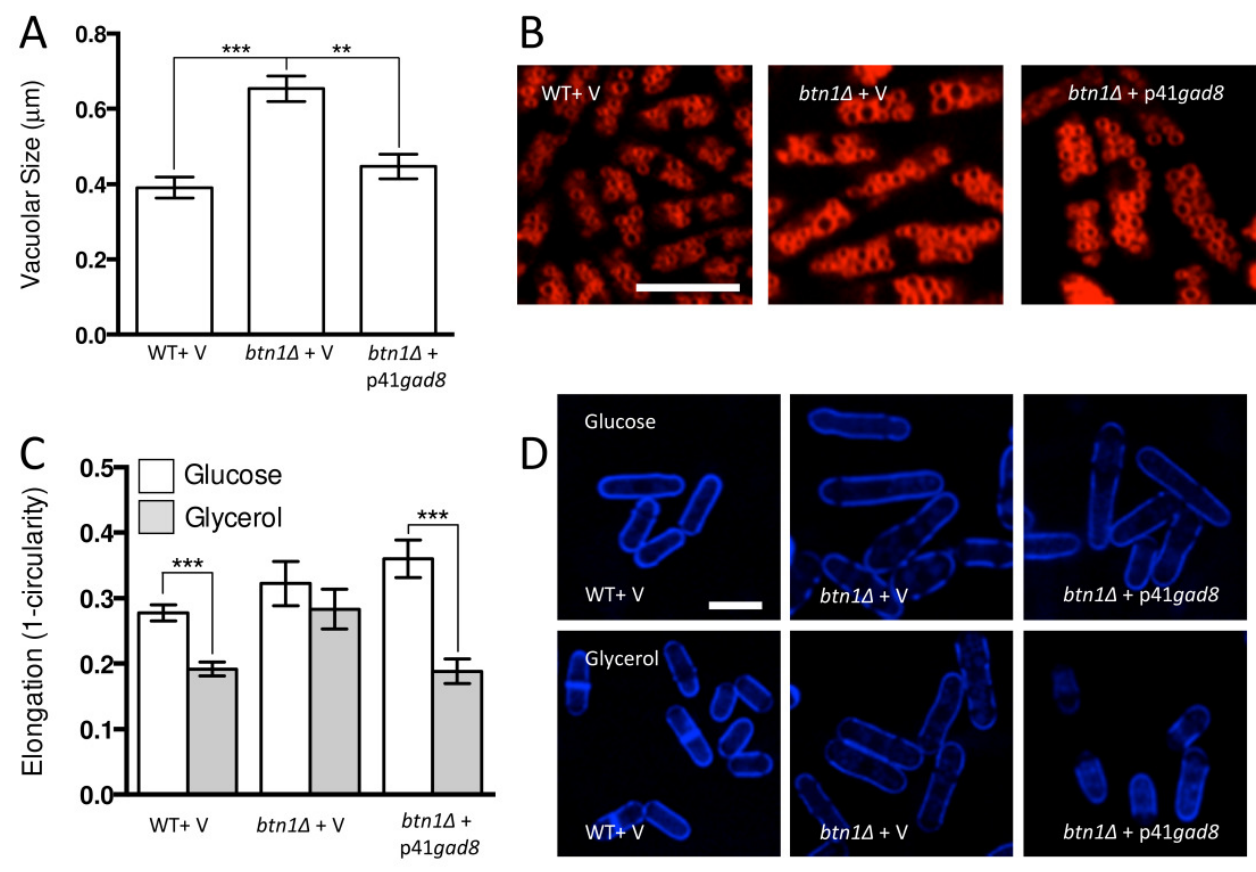

E
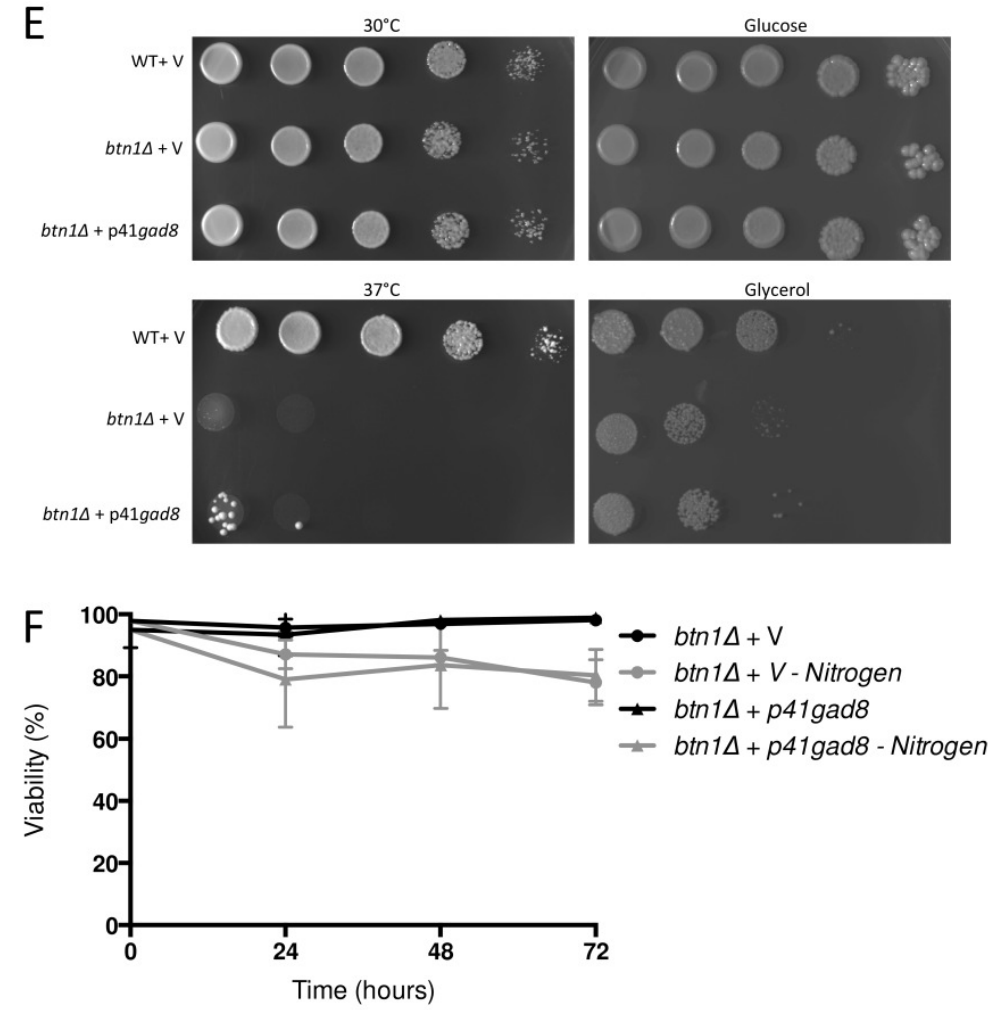

FIGURE 3: Increasing TORC2-dependent signalling rescues aspects of $b t n 1 \Delta$ phenotype. (A) Cells lacking btn1 were transformed with an expression vector containing gad8. Vacuole size was measured in these cells, in addition to WT and btn1 $1 \Delta$ cells containing empty vector, following staining with the vital dye FM4-64. The diameter of 300 vacuoles was determined per data set, and data shown is a mean ( \pm SEM) of 4 independent experiments. Statistical analysis was performed using a one-way ANOVA with a Tukey's multiple comparison post-test $\left(^{* *}=\mathrm{P}<0.01, * * *=\mathrm{P}<\right.$ 0.001). (B) Representative images of experiments as performed in (A) are shown. Scale bar represents $10 \mu \mathrm{m}$. (C) The morphological response of these populations to growth on glycerol was analysed following 6 hours in culture using a measure of cell elongation, on a scale of 0 to 1 , where 0 represents a perfectly round cell $\left(1-\left(\frac{4 \pi \text { area }}{\text { perimeter }^{2}}\right)\right)$. Data shown is a mean ( \pm SEM) of 5 independent experiments. Statistical significance between each condition was determined using a one-way ANOVA with a Tukey's multiple comparison post-test $(* * *=\mathrm{P}<0.001)$. (D) Representative images of experiments as performed in (C) are shown. Scale bar represents $10 \mu \mathrm{m}$. (E) These cells were serially diluted from a log-phase culture $\left(1 \times 10^{6}\right.$ cells/ml) and spotted onto YES plates. Plates were then incubated at $30^{\circ} \mathrm{C}$ or $37^{\circ} \mathrm{C}$ for 3 - 4 days to determine growth at high temperature.

They were also spotted onto plates containing either glucose or glycerol as a carbon source. Plates were then incubated at $30^{\circ} \mathrm{C}$ for $6-7$ days to determine growth under non-fermentative conditions. Images are representative of three independent experiments. (F) Viability upon nitrogen limitation was determined over periods of up to 72 hours in these cell populations, using propidium iodide to stain dead cells and calcofluor white to stain all cells. Cells were cultured in either $\mathrm{MM}$ or $\mathrm{MM}$ lacking a nitrogen source $\left(\mathrm{NH}_{4} \mathrm{Cl}\right)$. 500 cells were scored for viability per data set, and data shown is a mean ( \pm SEM) of 3 independent experiments. 
btn $1 \Delta$ cells.

TORC2 and CWI pathway: modulating distinct signalling nodes elicit different levels of correction in btn1 $\Delta$ cells Given the negative genetic interaction between btn1 and tor1, increasing TORC2 activity could alleviate aspects of the $b t n 1 \Delta$ phenotype. We initially explored this approach through overexpression of the downstream kinase Gad8, which is a multicopy suppressor of aspects of the loss of TORC2 [29]. A key phenotype in cells lacking btn1, and one that is strongly linked to the phenotype of CLN3 disease, is a change in vacuole homeostasis [12]. One of the clearest manifestations of this feature in btn $1 \Delta$ cells is an increase in vacuole size [12]. We investigated vacuole size in wildtype and $b t n 1 \Delta$ cells containing empty vector alone (as a control), as well as btn1 1 cells expressing gad8 from the $n m t 1$ promoter of the pREP41 plasmid (Fig. $3 \mathrm{~A}$ and B). Consistent with previous reports, btn1 $1 \Delta$ cells exhibited significantly larger vacuoles than wild-type cells $(0.65 \pm$ $0.03 \mu \mathrm{m}$ compared to $0.39 \pm 0.01 \mu \mathrm{m}, \mathrm{P} \leq 0.001)$. The overexpression of gad8 in btn $1 \Delta$ cells significantly rescued vacuole size $(0.40 \pm 0.03 \mu \mathrm{m})(P \leq 0.01)$, restoring them to near that of wild-type. These data provide the first indication that increasing TORC2 function significantly rescues a disease-relevant phenotype of $b \operatorname{tn} 1 \Delta$ cells.

As discussed above, the ability of cells to respond to glucose-limitation with a change in cell size requires TORC2 function [26]. To investigate whether the inability of btn1 $\Delta$ cells to undergo this morphological response can be rescued by increasing TORC2 pathway activity, we assessed the elongation of btn $1 \Delta$ cells overexpressing gad8 following growth for 6 hours in media containing either glucose or glycerol as a carbon source (Fig. $3 C$ and D). Wild-type and $b t n 1 \Delta$ cells containing empty vector alone displayed comparable responses to cells without vector (WT glucose $-0.28 \pm 0.01, \mathrm{WT}$ glycerol $-0.19 \pm 0.01, \mathrm{P} \leq 0.001 ;$ btn $1 \Delta$ glucose $-0.32 \pm 0.02, b t n 1 \Delta$ glycerol $-0.28 \pm 0.01, n s)$. The overexpression of gad8 was able to significantly rescue this morphological response, despite these cells being elongated under growth in glucose (glucose $-0.36 \pm 0.01$, glycerol $0.19 \pm 0.01, P \leq 0.001)$.

Despite this rescue, expression of gad8 was unable to significantly rescue the growth of btn $1 \Delta$ cells on glycerol or at high temperature, suggesting that the ability of these cells to adapt to heat and nutrient stress is still impaired (Fig. 3E). Similarly, expression of gad8 was unable to rescue the viability of $b \operatorname{tn} 1 \Delta$ cells grown in the absence of a nitrogen source (Fig. 3F).

Given that the phenotypes not rescued by increasing TORC2 activity were linked to stress and nutrient adaptation, we reasoned that modulating processes more closely linked to the CWI pathway might be more effective in correcting these phenotypes. These processes seem particularly relevant in this instance, as this pathway is known to respond to both heat and glucose limitation. In particular, mutants of the pmk1 MAP kinase, like btn1 $\Delta$ mutants, display a partial growth defect on glycerol. Further, this pathway is required for proper activation of Sty1 (the MAP kinase of the SAPK pathway) under glucose-limitation, inte- grating these processes with further identified btn1 interactors [30].

To increase CWI activity, we chose to increase Rho GTPase levels in these cells, which represent a key hub in CWI pathway regulation, by overexpressing the btn 1 interactor rho5 and its essential paralogue rho1. Overexpression of rho1 significantly decreased vacuole size in btn1 $\Delta$ cells $(P \leq 0.001)$ to near that of wild-type cells containing empty vector alone (WT $-0.30 \pm 0.02 \mu \mathrm{m}$, btn $1 \Delta-0.47 \pm$ $0.04 \mu \mathrm{m}, b t n 1 \Delta$ with p41rho1 - $0.32 \pm 0.01 \mu \mathrm{m}$ ) (Fig. 4A and $B)$. The overexpression of rho5 led to an over-correction of this phenotype $(0.19 \pm 0.01 \mu \mathrm{m})$, producing vacuoles significantly smaller than those of $b \operatorname{tn} 1 \Delta$ cells $(P \leq 0.001)$ and wild-type cells containing vector alone $(P \leq 0.05)$. Consistent with these observations, over-expression of $p m p 1$, which inhibits the activity of pmk1 [31], caused an increase in vacuole size in $b t n 1 \Delta$ cells $(b t n 1 \Delta$ with p41pmp1- $0.80 \pm$ $0.08 \mu \mathrm{m}$ vs btn1 $\Delta-0.52 \pm 0.04 \mu \mathrm{m}, \mathrm{P} \leq 0.01$ ) but not in wild-type cells (WT with p41pmp1-0.24 $\pm 0.01 \mu \mathrm{m}$ vs WT $-0.23 \pm 0.02, \mathrm{~ns}$ ) (Fig. 4C). Such data indicate that increasing CWI pathway activity is able to correct the vacuole defect of $b \operatorname{tn} 1 \Delta$ cells, and directly inhibiting this pathway exacerbates the vacuole defect. The over-correction by rho5 could indicate a strong dose-dependence in this particular rescue. Indeed, previous work has demonstrated such dose dependence in the relationship between btn 1 and vacuole size [12].

We next investigated whether the Rho GTPases were able to rescue the morphological response of these cells to glycerol, as seen with gad8. We looked just at rho1 in this case, as rho5 overexpression has a pronounced effect on cell shape and septation [32], making morphological comparisons difficult (Fig. S2). Overexpression of rho1 elicited a rescue of this response, with cells displaying a significant reduction in cell elongation when grown in glycerol containing media $(0.28 \pm 0.02$ to $0.16 \pm 0.01, P \leq 0.01)$ (Fig. $4 \mathrm{D}$ and $\mathrm{E})$.

Further to a rescue of the morphological response to glycerol, both rho1 and rho5 were able to correct the growth defect of btn1 $1 \Delta$ cells on glycerol as a carbon source. In addition, rho1 was able to rescue the temperature sensitivity phenotype of these cells (Fig. 4F). The overexpression of rho5 did not correct this phenotype, although, given the link between heat-sensitivity and septation defects in btn $1 \Delta$ cells [15] and the enhanced septation defect of cells overexpressing rho5 (Fig. S2), this is not wholly unexpected.

In addition to these phenotypes, overexpression of rho 1 and rho5 rescued the viability of btn $1 \Delta$ cells when grown in the absence of a nitrogen source, with viability remaining higher than $95 \%$ throughout the course of the experiment (Fig. 4G). These data provide evidence for a profound positive interaction between CWI pathway signalling and btn1, indicating that such processes are intimately linked with the defects that occur in btn1 $\Delta$ cells.

\section{Reducing TORC1 activity corrects defects in btn $1 \Delta$ cells} TORC1 activity is a key signal of a favourable environment, promoting proliferation and suppressing stress-responsive 


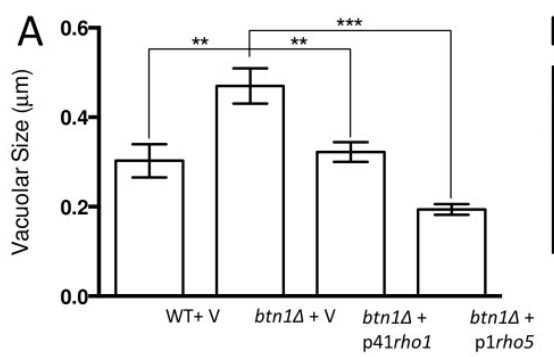

B
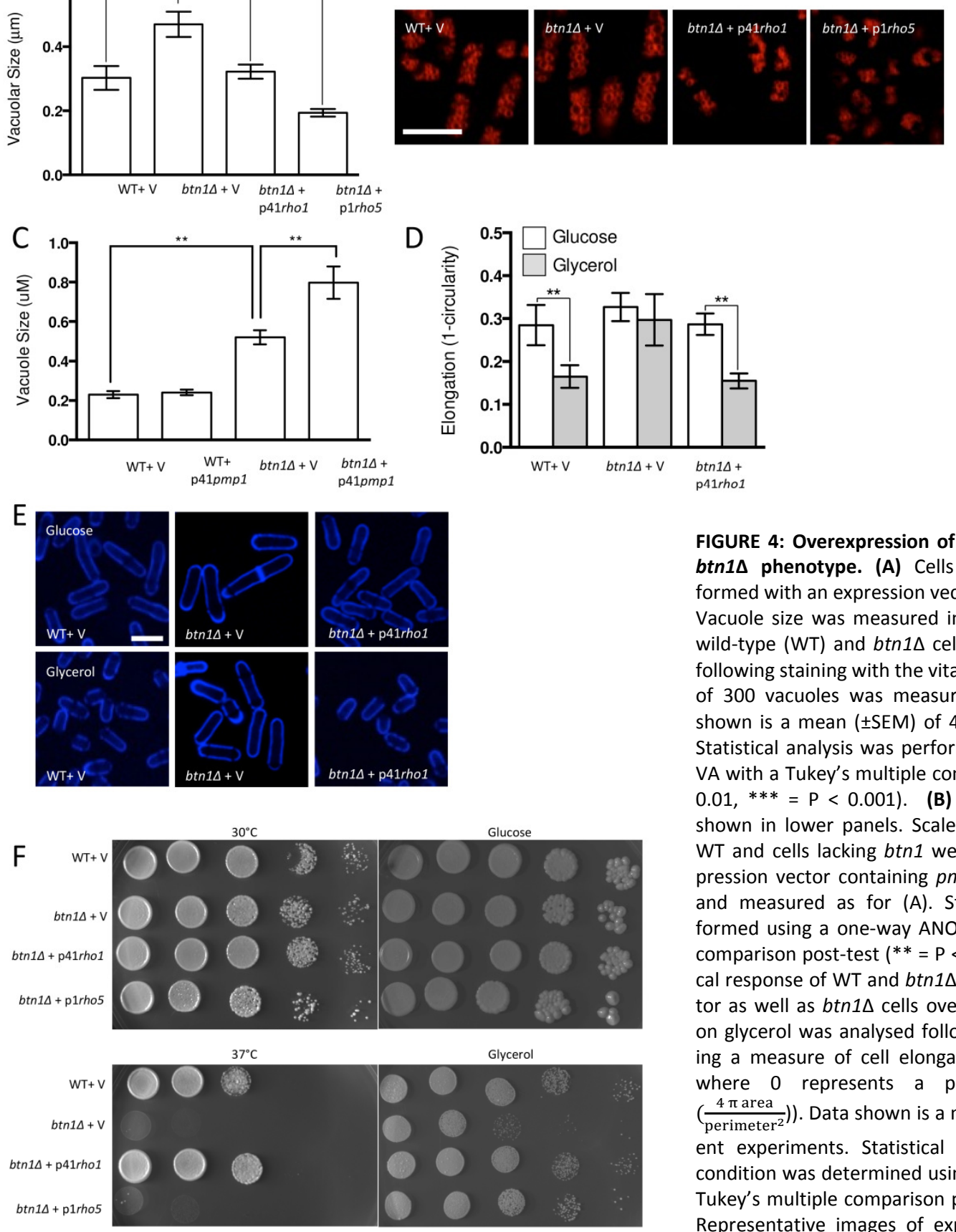

FIGURE 4: Overexpression of Rho rescues all aspects of btn1s phenotype. (A) Cells lacking btn1 were transformed with an expression vector containing rho1 or rho5. Vacuole size was measured in these cells, in addition to wild-type (WT) and btn1 $1 \Delta$ cells containing empty vector, following staining with the vital dye FM4-64. The diameter of 300 vacuoles was measured per data set, and data shown is a mean ( \pm SEM) of 4 independent experiments. Statistical analysis was performed using a one-way ANO$V A$ with a Tukey's multiple comparison post-test $\left({ }^{* *}=\mathrm{P}<\right.$ $0.01, * * *=\mathrm{P}<0.001$ ). (B) Representative images are shown in lower panels. Scale bar represents $10 \mu \mathrm{m}$. (C) WT and cells lacking btn1 were transformed with an expression vector containing pmp1. Vacuoles were stained and measured as for (A). Statistical analysis was performed using a one-way ANOVA with a Tukey's multiple comparison post-test ( $\left.{ }^{* *}=\mathrm{P}<0.01\right)$. (D) The morphological response of WT and $b t n 1 \Delta$ cells containing empty vector as well as $b t n 1 \Delta$ cells overexpressing rho1 to growth on glycerol was analysed following 6 hours in culture using a measure of cell elongation, on a scale of 0 to 1 , where 0 represents a perfectly round cell (1$\left(\frac{4 \pi \text { area }}{\text { perimeter }}{ }^{2}\right)$ ). Data shown is a mean ( \pm SEM) of 5 independent experiments. Statistical significance between each condition was determined using a one-way ANOVA with a Tukey's multiple comparison post-test $\left({ }^{* *}=\mathrm{P}<0.01\right)$. (E) Representative images of experiments as performed in (D) are shown. Scale bar represents $10 \mu \mathrm{m}$. (F) Wild-type and $b t n 1 \Delta$ cells containing empty vector as well as $b t n 1 \Delta$ cells overexpressing rho 1 and $r$ o5 5 were serially diluted from a log-phase culture $\left(1 \times 10^{6}\right.$ cells $\left./ \mathrm{ml}\right)$ and spotted onto YES plates. Plates were then incubated at $30^{\circ} \mathrm{C}$ or $37^{\circ} \mathrm{C}$ for 3-4 days to determine growth at high temperature. They were also spotted onto plates containing either glucose or glycerol as a carbon source. Plates were then incubated at $30^{\circ} \mathrm{C}$ for $6-7$ days to determine growth under non-fermentative conditions. Images are representative of three independent experiments. (G) Viability upon nitrogen limitation was determined over periods of up to 72 hours in these cell populations, using propidium iodide to stain dead cells and calcofluor white to stain all cells. Cells were cultured in either $\mathrm{MM}$ or $\mathrm{MM}$ lacking a nitrogen source $\left(\mathrm{NH}_{4} \mathrm{Cl}\right)$. 500 cells were scored for viability per data set, and data shown is a mean ( $\pm \mathrm{SEM}$ ) of 3 independent experiments. 
processes [24]. As a consequence, it is antagonistic to many of the processes explored in this study that improved the btn1 $1 \Delta$ phenotype. TORC1 represses TORC2 function [23], and negatively regulates Sty1 [33], which displays extensive cross-talk with the CWI pathway [30]. This relationship is highlighted by the fact that the core TORC1 component gene tco89 is a positive interactor of btn1.

In order to test whether repression of TORC1 activity could rescue the phenotypes of btn $1 \Delta$ cells, we first overexpressed a dominant-negative form of the upstream activator of TORC1, Rhb1 ( $\left.r h b 1^{D 60 K}\right)$ [34]. Upon overexpression of dominant-negative rhb1 (dnrhb1) in btn1 $\Delta$ cells, we observed a significant reduction $(P \leq 0.001)$ in vacuole size compared to btn $1 \Delta$ cells containing vector alone (btn $1 \Delta$ with $\mathrm{p} 41 d n r h b 1-0.40 \pm 0.04 \mu \mathrm{m}, b \operatorname{tn} 1 \Delta$ with empty vector
$-0.65 \pm 0.03 \mu \mathrm{m})$ (Fig. 5A and B). This result was comparable to the vacuole size of wild-type cells $(0.39 \pm 0.01 \mu \mathrm{m})$, indicating a rescue of vacuole morphology upon repression of TORC1 activity.

We next investigated the ability of TORC1 repression to rescue the nutrient-sensing defects of cells lacking btn 1 . The overexpression of $d n r h b 1$ led to a significant rescue of the morphological response of these cells to glucoselimitation, following 6 hours growth in glycerol as a carbon source (Fig. $5 C$ and D). Cell elongation fell from $0.29 \pm 0.02$ to $0.18 \pm 0.01$ when grown in glycerol as opposed to glucose, a response comparable to that observed in wild-type cells (glucose $-0.28 \pm 0.01$, glycerol $-0.19 \pm 0.01$ ). This also corresponded to a rescue of the growth defect of these cells on glycerol (Fig. 5E). Further to the glycerol growth
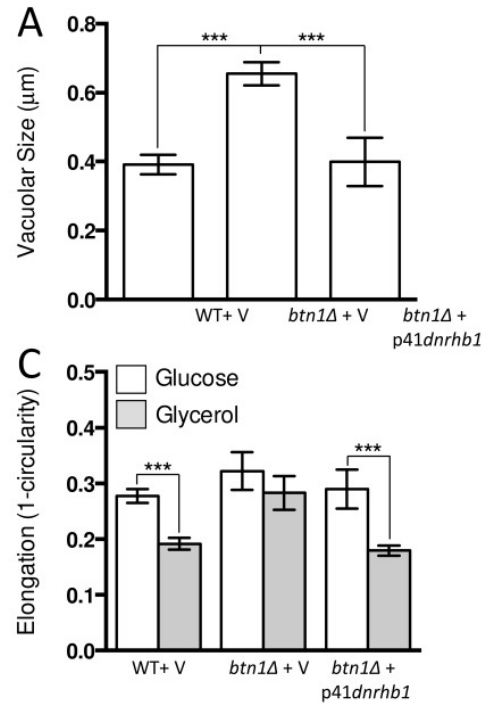

B

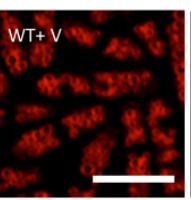

D
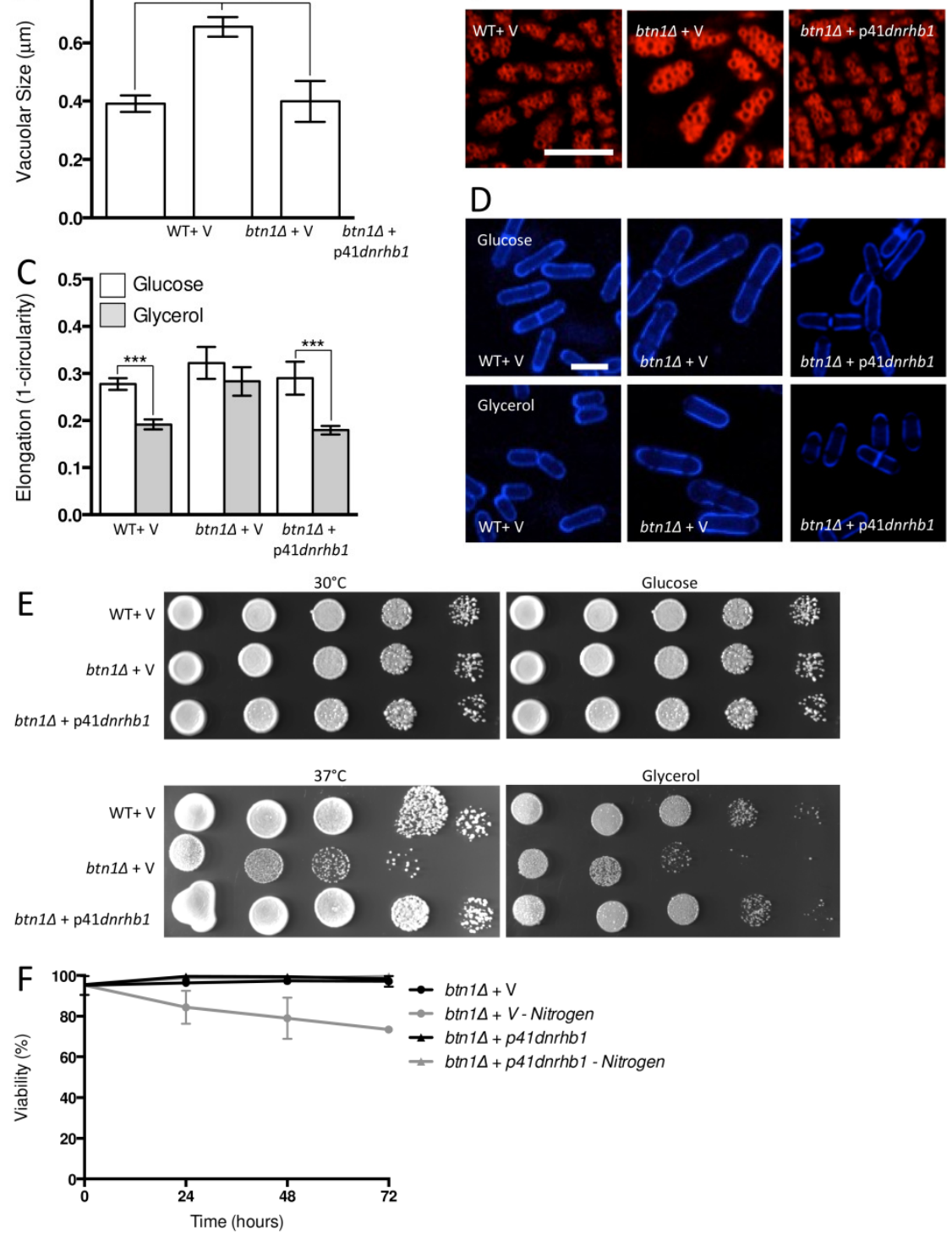

FIGURE 5: Inhibiting TORC1 function rescues the defects in btn1s cells. (A) Cells lacking btn1 were transformed with an expression vector containing a dominantnegative form of rhb1 (dnrhb1). Vacuole size was measured in these cells, in addition to wild-type (WT) and btn1 $\Delta$ cells containing empty vector, following staining with the vital dye FM4-64. The diameter of 300 vacuoles was measured per data set, and data shown is a mean ( \pm SEM) of 4 independent experiments. Statistical analysis was performed using a one-way ANOVA with a Tukey's multiple comparison post-test $(* * *=\mathrm{P}$ $<0.001)$. (B) Representative images of experiments as performed in (A) shown. Scale bar represents $10 \mu \mathrm{m}$. (C) The morphological response of these populations to growth on glycerol was analysed following 6 hours in culture using a measure of cell elongation, on a scale of 0 to 1 , where 0 represents a perfectly round cell $\left(1-\left(\frac{4 \pi \text { area }}{\text { perimeter }^{2}}\right)\right)$. Data shown is a mean ( \pm SEM) of 5 independent experiments. Statistical significance between each condition was determined using a one-way ANOVA with a Tukey's multiple comparison post-test $(* * *=\mathrm{P}<$ 0.001). (D) Representative images of experiments as performed in (C) shown. Scale bar represents $10 \mu \mathrm{m}$. (E) These cells were serially diluted from a log-phase culture $\left(1 \times 10^{6}\right.$ cells $/ \mathrm{ml}$ ) and spotted onto YES plates. Plates were then incubated at $30^{\circ} \mathrm{C}$ or $37^{\circ} \mathrm{C}$ for $3-4$ days to determine growth at high temperature. They were also spotted onto plates containing either glucose or glycerol as a carbon source. Plates were then incubated at $30^{\circ} \mathrm{C}$ for $6-7$ days to determine growth under non-fermentative conditions. Images are representative of three independent experiments. (F) Viability upon nitrogen limitation was determined over periods of up to 72 hours in these cell populations,
tured in either MM or MM lacking a nitrogen using propidium iodide to stain dead cells and calcofluor white to stain all cells. Cells were cultured in either MM or MM lacking a nitrogen source $\left(\mathrm{NH}_{4} \mathrm{Cl}\right) .500$ cells were scored for viability per data set, and data shown is a mean ( \pm SEM) of 3 independent experiments. Scale bar represents $10 \mu \mathrm{m}$. 
defect, dnrhb1 overexpression also rescued the heat sensitivity of btn $1 \Delta$ cells (Fig. 5E). Finally, this construct was also able to elicit a complete rescue of viability under nitrogen limiting conditions, with viability remaining above $95 \%$ throughout the course of the experiment (Fig. 5F).

Given the substantial rescue of btn1 $1 \Delta$ cells by dnrhb1mediated activation of TORC1, we wanted to confirm the interaction between btn1 and TORC1 by another means. We chose to use two pharmacological inhibitors of TORC1, rapamycin (allosteric) and caffeine (competitive inhibitor of ATP binding) [35]. TORC1 antagonism leads to cell rounding a response that mimics the morphological change ordinarily exhibited by wild-type cells in response to nitrogen and amino acid starvation. Therefore, we examined the effect of TORC1 antagonism on cell rounding [36] and on vacuole size, heat sensitivity, and growth in glycerol. As expected, wild-type cells displayed a significant reduction in cell elongation upon rapamycin treatment for 6 hours $(0.26 \pm$ 0.02 to $0.15 \pm 0.01, \mathrm{P} \leq 0.01$ ) (Fig. $6 \mathrm{~A}$ and $\mathrm{B}$ ). This reduction in cell elongation did not change further upon exposure to both rapamycin and caffeine [35]. Cells lacking btn1, however, did not respond to rapamycin, displaying only a slight morphological change $(0.30 \pm 0.01$ to $0.25 \pm 0.02)$ that was slightly enhanced by the addition of caffeine and rapamycin, but again not significantly (0.22 \pm 0.03$)$ (Fig. 6A and B). The addition of caffeine and rapamycin to wild-type cells did not significantly affect vacuole size (vehicle - $0.37 \pm$ $0.05 \mu \mathrm{m}$, rapamycin - $0.31 \pm 0.03$, caffeine and rapamycin $0.24 \pm 0.01$ ) (Fig. $6 C$ and D). Rapamycin alone had no effect on the vacuole size of cells lacking btn1, but, in contrast, a combination of caffeine and rapamycin significantly $(\mathrm{P} \leq$ 0.001 ) reduced vacuole size (vehicle $-0.58 \pm 0.03 \mu \mathrm{m}$, rapamycin - $0.55 \pm 0.04$, caffeine and rapamycin $0.30 \pm 0.02$ ) (Fig. 6C and D). Further, treatment with rapamycin alone elicited a rescue of both the heat sensitivity defect and glycerol growth defect of cells lacking btn1 (Fig. 6E). The ability of these pharmacological treatments to rescue the defects in vacuole size, heat sensitivity and growth in glycerol of btn1 $1 \Delta$ cells appears to be closely linked to their ability to target TORC1 signalling itself, as opposed to changes
A
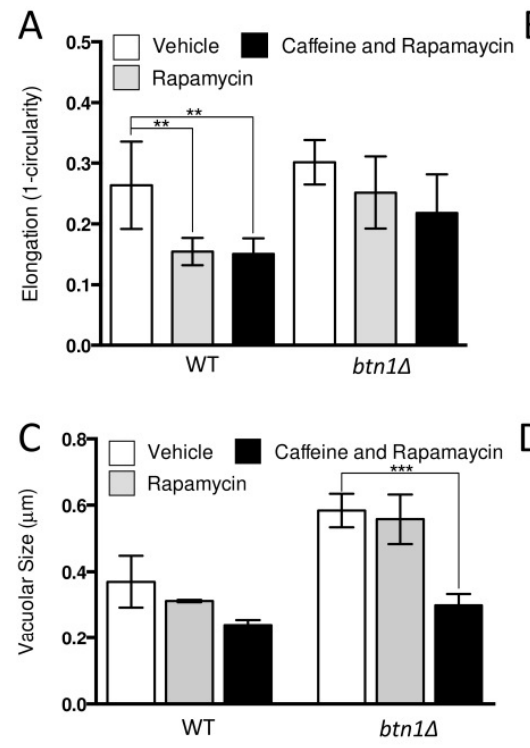

$E$

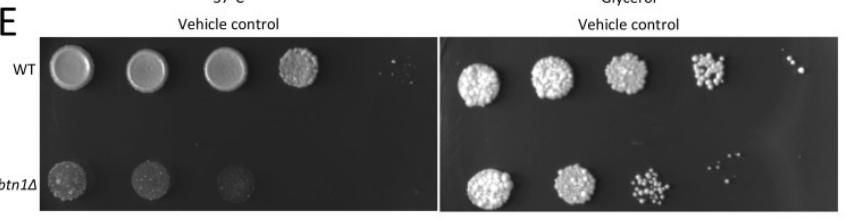

100ug/ml Rapamycin

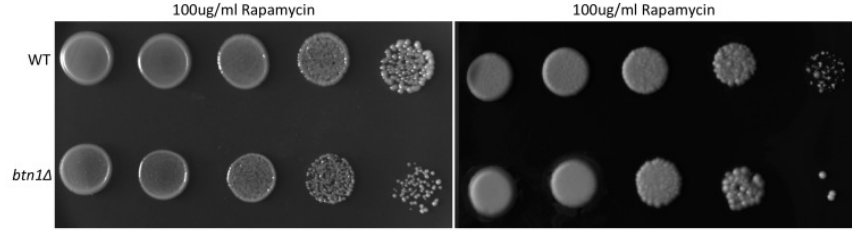

B
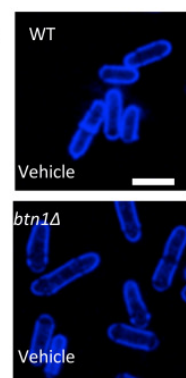

D
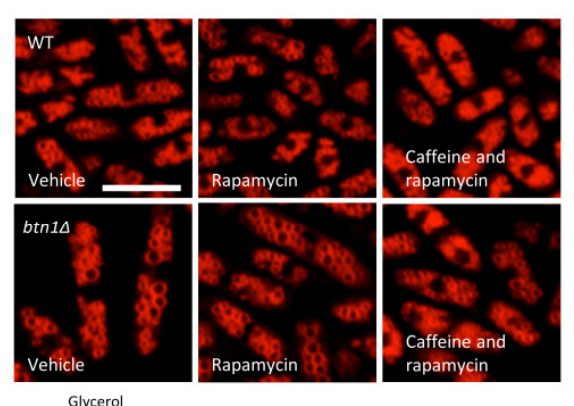

rapamycin

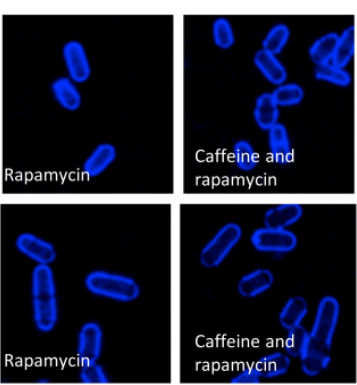

FIGURE 6: Pharmacological inhibition of TORC1 function replicates rescue by overexpression of dominant-negative rhb1. (A) The morphological response of wild-type (WT) and btn1s cells to the TORC1 antagonists rapamycin $(100 \mu \mathrm{g} / \mathrm{ml})$ and caffeine and rapamycin combined (10 $\mathrm{mM}$ and $100 \mu \mathrm{g} / \mathrm{ml}$ respectively) was analysed following 6 hours in culture using a measure of cell elongation, on a scale of 0 to 1 , where 0 represents a perfectly round cell $\left(1-\left(\frac{4 \pi \text { area }}{\text { perimeter }}\right)\right)$. Data shown is a mean ( \pm SEM) of 6 independent experiments. Statistical significance between each condition was determined using a one-way ANOVA with a Tukey's multiple comparison post-test $(* *=\mathrm{P}<$ 0.01). (B) Representative images of experiments as performed in (A) are shown. Scale bar represents $10 \mu \mathrm{m}$. (C) Wild-type and $b$ tn $1 \Delta$ cells were grown for 6 hours in $\mathrm{MM}$ alone or with the TORC1 antagonists rapamycin $(100 \mu \mathrm{g} / \mathrm{ml})$ and caffeine and rapamycin $(10 \mathrm{mM}$ and $100 \mu \mathrm{g} / \mathrm{ml}$ respectively). Vacuole size was then measured in these cells following staining with the vital dye FM4-64. The diameter of 300 vacuoles was measured per data set, and data shown is a mean ( \pm SEM) of 4 independent experiments. Statistical analysis was performed using a one-way ANOVA with a Tukey's multiple comparison post-test $\left({ }^{* * *}=\mathrm{P}<0.001\right)$. (D) Representative images of experiments as performed in (C) are shown. Scale bar represents $10 \mu \mathrm{m}$. (E) WT and btn $1 \Delta$ cells were serially diluted from a log-phase culture $\left(1 \times 10^{6}\right.$ cells $/ \mathrm{ml})$ and spotted onto YES plates either containing or lacking rapamycin $(100 \mu \mathrm{g} / \mathrm{ml})$. Plates were then incubated at $37^{\circ} \mathrm{C}$ for $3-4$ days to determine growth at high temperature. They were also spotted onto plates containing glycerol as a carbon source either containing or lacking rapamycin $(100 \mu \mathrm{g} / \mathrm{ml})$. Plates were then incubated at $30^{\circ} \mathrm{C}$ for $6-7$ days to determine growth under non-fermentative conditions. Images are representative of three independent experiments. 
in the activity of the connected SAPK pathway, as expression of a constitutively active wis1 mutant (wis1DD) [37] did not rescue these phenotypes (Fig S3A - C). These data confirm that activation of the TORC1 pathway pharmacologically in btn1 $1 \Delta$ cells rescues most of the phenotypes arising from loss of btn 1 function. However, the pharmacological inhibition of the TORC1 pathway is not completely equivalent to the inhibition of the TORC1 pathway using dnrhb1 in btn $1 \Delta$ cells.

\section{DISCUSSION}

In this study, SGA analysis was used as an unbiased approach to identify the genetic interactors of btn 1 , and place btn 1 into its biological context within the whole cell. This approach highlighted a key role for the Tor kinase complexes, TORC1 and TORC2, in the dysfunctional changes that occur in fission yeast lacking btn1, as one third of the interactions connected directly with pathways in which these complexes are active. Further, we have shown that $b t n 1 \Delta$ cells display defects in their stress response to nitrogen and glucose limitation, in addition to osmoremedial heat sensitivity, consistent with TORC1 and TORC2 dysfunction in these cells (Fig. 7).
Specifically, the SGA identified negative interactions in tor 1 , the core kinase component of TORC2, multiple components of the stress-activated protein kinase (SAPK) pathway, and a component of the linked CWI pathway. In addition, tco89, encoding a core component of TORC1, which antagonises both the SAPK cascade and TORC2 signalling, was identified as a positive genetic interactor of btn1. btn1 was also found to interact negatively with a number of genes that display stress-responsive expression.

TORC1 negatively regulates a number of processes associated with cellular catabolism and adaptation to stress [24]. One of these roles is in the repression of autophagy. Autophagy similarly appears important to the fitness of btn $1 \Delta$ cells, as atg14 and atg12 were identified as negative interactors of btn1, and both are required for autophagosome formation [38]. In support of this work, and the use of yeast as a model, defective autophagy has previously been linked to CLN3 disease [39]. In repressing stressresponsive processes, TORC1 is known to negatively regulate sty 1 , the mitogen-activated protein (MAP) kinase and component of the stress-activated protein kinase (SAPK) cascade [33]. Both wis4 and wis1, encoding the MAP kinase kinase kinase and MAP kinase kinase of the SAPK pathway,

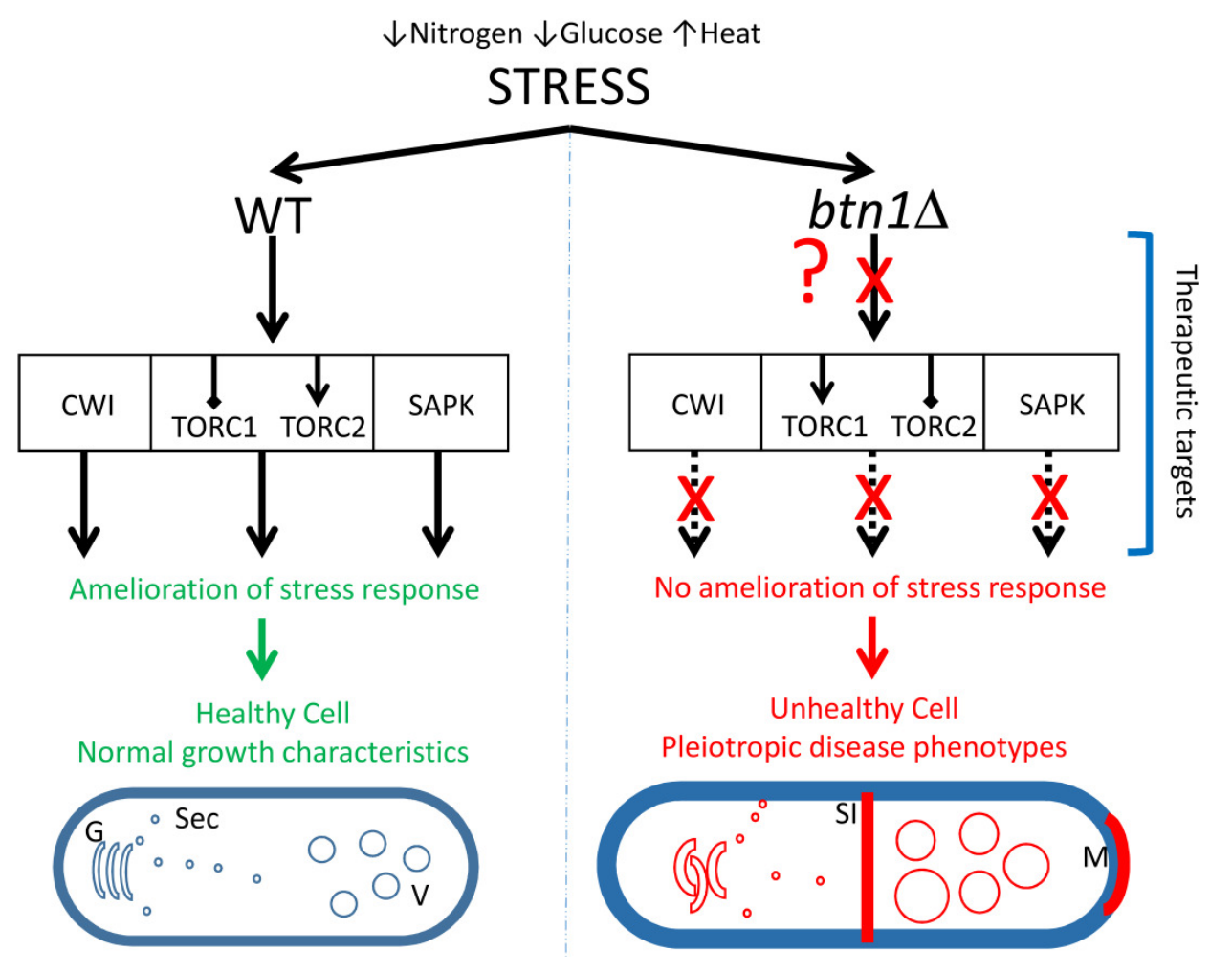

FIGURE 7: Diagrammatic summary of the effect of loss of btn1 on the response to stress. The left panel shows the response of healthy cells to stresses applied in this study, such as low nitrogen or glucose or raised temperature. These cells are able to respond via interconnected signaling pathways such as CWI, TORC and SAPK. Activation of these pathways allows the cell to respond to the effects of stress and restore cell homeostasis and integrity. In contrast, cells lacking btn1 are unable to respond to the applied stresses (represented by '?' and ' $X$ ') and undergo a variety of previously reported responses that include an increase in vacuole size, swollen and disorganized Golgi and mistrafficking of vacuole enzymes such as carboxypeptidase $Y$, increased septation index and longer cell cycle and cell length, monopolar growth, defective cell wall, absence of cell rounding (in response to low glucose). Genetic or pharmacological activation of the three signalling pathway provides partial or full rescue of these pleiotropic phenotypes of btn1 $1 \Delta$ cells. G, Golgi; Sec, secretory vesicles; V, vacuole; SI, division septum; $\mathrm{M}$, monopolar growth. 
respectively, interact negatively with $b t n 1$, as does the transcription factor gene pap1, which is a downstream target of sty1 [40]. Furthermore, btn1 interacts negatively with a number of genes that display stress-responsive expression.

TORC2 is a positive regulator of stress-responsive processes [29], and is also involved in cytoskeletal organization, vacuolar morphology and glucose sensing [22]. In addition to a negative interaction with the gene encoding the Tor kinase component of TORC2 (tor1), btn1 also negatively interacts with genes encoding components of the connected CWI MAP kinase cascade. The relationship between TORC2 and the CWI pathway is complex. TORC2 is required for the response to cell wall stressors [41], and is a direct regulator of the CWI pathway through the activation of the Rho guanine-nucleotide exchange factor (GEF) ROM2 in budding yeast [42]. In fission yeast, it has recently been shown that Tor1, acting as part of TORC2, enhances Pck2 synthesis to activate the $\mathrm{CWI}$ under both cell wall stress and glucose limitation conditions [43].

Despite this positive relationship, recent work in fission yeast has indicated a negative regulation of TORC2 by pmk1, the MAP kinase component of the fission yeast CWI pathway, at least in certain conditions [25]. In fission yeast both Rho1 and Rho2 are well established as regulators of the CWI pathway [44, 45]. Rho5 acts as a functional homologue of rho1, sharing the role of regulating cell wall homeostasis [46]. More recently, rho5 has also been shown to be an upstream regulator of the CWI [47]. The regulation of this pathway is complex as Rho1 both positively [45] and negatively [48] regulates the downstream MAP kinase cascade. This potentially intricate relationship is reflected in the interactors of btn1 that fall within this pathway, that exhibit both positive and negative effects upon signalling. btn1 exhibits a negative interaction with the rho1 homologue gene rho5, and a number of genes involved in downstream cytoskeletal and polarity components. Despite this, the Rho GEF gene $r g f 1$, which is a positive regulator of the CWI pathway [49], is a positive interactor of $b t n 1$ whereas $r d i 1$ (encoding a GDP dissociation factor) is a negative interactor. This multifaceted pattern of interactions is consistent with current understanding of this pathway, given the complex interactions between these signalling nodes.

Although a process that maintains CWI might seem fungal-specific, and not relevant to disease in a higher eukaryote, previous work in budding yeast revealed similar dysfunctional processes in cells overexpressing $\alpha$-synuclein as a model for Parkinson's disease, suggesting that there is a mammalian counterpart to the CWI pathway in yeast. Such observations are of specific relevance to CLN3 disease, as $\alpha$-synuclein is also upregulated in this condition [9]. RHO1 signalling and the CWI pathway are disrupted by overexpression of $\alpha$-synuclein [50]. Further, it was demonstrated that similar changes in downstream kinase cascades could be observed in mammalian cells overexpressing $\alpha$-synuclein. Affected kinases included c-Jun, which has previously been linked to CLN3 disease, and is part of a pathway closely related to the SAPK pathway in yeast [51].
This is not the only instance in which Rho signalling has been linked to neurodegeneration. Leucine-rich repeat kinase 2 (LRRK2), mutations in which represent the most common cause of familial late-onset Parkinson's disease, has been shown to bind Rac1, and to a lesser extent RhoA and Cdc42 [52]. All of these binding partners are members of the Rho GTPase family. The binding of LRRK2 to Rac1 led to its activation and relocalisation, and was lost when disease-causing mutations were introduced into LRRK2. Further, the overexpression of Rac1 was shown to be protective in these cells. Other studies have also demonstrated a protective effect of Rho signalling, focusing both on Rho GEFs [53] and the Rho GTPases themselves [54]. Such studies highlight how processes as seemingly specialized as CWI in yeast can be highly informative of processes relevant to disease in higher order eukaryotes. Further, they demonstrate that Rho-dependent signalling could be a vital neuroprotective process in more common neurodegenerative disease, as well as being a key pathway in the neurodegenerative disease model presented in this study.

Importantly, the validation of these interactions during this work revealed interventions that produced a complete rescue of all phenotypes investigated in the btn1 $1 \Delta$ strain, including phenotypes linked to TORC1 function, TORC2 function, CWI and vacuole homeostasis. Among the interactions investigated, increasing Rho1 levels, and thereby upregulating the main signalling node in the CWI pathway, or repressing TORC1 function (pharmacologically or genetically) led to complete rescues.

More notably, a neuroprotective role of TORC1 repression is already well established in a number of different systems [55]. A protective effect of the TORC1 antagonist rapamycin has been demonstrated in models for Parkinson's disease [57], Huntington's disease [56], spinoceribellar ataxia type 3 [58] and retinal degeneration mediated by mitochondrial dysfunction [59]. In addition, Tor signalling is also elevated in Alzheimer's disease models [60].

It is important to note, however, that targeting TORC1 in disease presents some technical challenges. Although in some studies an increase in lysosome function has been observed upon rapamycin treatment [61], other work indicates that rapamycin is an incomplete antagonist of TORC1 and a poor inducer of lysosomal functions [62], in concert with our observations. Another complication of targeting TORC1 in disease is the antagonistic role of TORC2. TORC2 signalling and the connected CWI pathway were both protective in our model for CLN3 disease. Further to the protective role of Rho signalling in a Parkinson's disease model [50], TORC2/AKT signalling has also been shown to be neuroprotective in a separate study of Parkinson's disease [63]. As a result, general Tor antagonists, such as Torin1, which target both TORC1 and TORC2, do not produce the same positive outcomes that TORC1 specific antagonists are able to elicit [57]. In addition, chronic rapamycin exposure also leads to a reduction in TORC2 activity. This fact could explain why rapamycin has been shown to be detrimental in certain studies, for example in one study using a fruit fly model for Alzheimer's disease [64]. 


\section{Conclusion}

The data presented here highlight a set of interconnected pathways that, when modulated correctly, produce a profound rescue of the dysfunctional changes observed in a yeast model for neurodegeneration caused by mutations in a single gene (Fig. 7). Links between CLN3 and the response to stress have previously been reported in other model organisms $[51,65]$, supporting the relevance of this yeast work to human CLN3 disease. Importantly however, a link between CLN3 and Tor signalling specifically, as reported here, is a novel mechanistic and therapeutic insight into CLN3 disease, the most common paediatric neurodegenerative disease. Furthermore, the pathways identified display strong parallels with known protective pathways in more common dementias, providing not only strong biological plausibility for the importance of these interactions, but also an indication that common processes may be exploited in therapeutic development for seemingly disparate neurodegenerative diseases.

\section{MATERIALS AND METHODS}

\section{Yeast strains and cell growth}

The strains used in this study were the wild-type strains SP23 (h-, ura4-D18, leu1-32, ade6-M210) and SP38 (h-, ura4-D18, leu1-32, ade6-210, his-) and btn1s strains SP29 (h-, btn1::NatMX, ura4-D18, leu1-32, ade6-210,) and SP35 (h-, btn1::NatMX, ura4-D18, leu1-32, ade6-210, his-). SP29 and SP35 were generated using the long primer method described in [66], using pFA6a-NatMX as a template [67]. Both strain backgrounds were used for SGA analysis (two isolates of each) to account for strain background differences, and SP23 and SP29 were used in all other analyses. Cell growth and manipulations were performed as described previously [68]. Minimal media (MM) was used in all experiments, unless otherwise stated. In nitrogen starvation experiments $\mathrm{NH}_{4} \mathrm{Cl}$ was omitted. Glucose and glycerol media were made as described previously [69]. Appropriate supplements were added to the media as required. All assays were performed using log-phase cultures $\left(1 \times 10^{6}\right.$ cells $\left./ \mathrm{ml}\right)$. The $d n r h b 1$ construct (pREP4X-rhb $1^{D 60 K}$ ) was obtained from Dr. Elizabeth Henske [34], and the gad8 construct (pREP41-gad8) from Dr. Ronit Weisman [70]. pREP1rho5 was obtained from Dr. Kentaro Nakano [46], pREP41rho1 from Dr. Pilar Pérez [48] and pREP1-wis1DD-3HA6His from Dr. Kaz Shiozaki [71]. The GFP-btn1 construct used in this study was described previously (pREP41-GFP-btn1) [12]. SP23 containing empty vector (pREP41) was used as a control in all experiments using these constructs.

\section{Synthetic genetic arrays (SGAs)}

SGA analysis was performed as described in [20], using a Singer RoToR robot (S. pombe settings) and the Bioneer haploid library (v2.0) and analysed as described previously [72]. A control SGA using ade6::NatMX4 as a query was performed simultaneously. The ade6 $\triangle$ mutant does not alter the fitness of the deletion collection, and arising double mutants are used to determine Bioneer strain fitness and for colony size normalisation. After germination, haploid double mutants were pinned to YES + G418 + Nat in a 1536 format. After three days growth at $30^{\circ} \mathrm{C}$, agar plates were imaged using a MultiDoc-lt Imaging System (UVP) and processed for analysis using the Gitter image analysis R package [73]. Using Excel, the colony sizes produced by Gitter were normalized to the median colony size of each plate to account for within-plate positional growth effects and differential plate growth. These normalized values were used to establish the ratio of query $b \operatorname{tn} 1 \Delta$ mutant size to control (ade6:NatMX4) colony size. Ratios from the four $b \operatorname{tn} 1 \Delta$ isolates used for the screen were used to calculate a mean ratio. Using this mean value, colony-size ratio scores of $<0.8$ were defined as negative interactions, and ratios of $>$ 1.2 as positive interactions. Genetic interactions were scored from two independent biological experiments. Only interactions common to both replicates were considered robust enough for follow-up. Negative and positive interaction sets were tested using PANTHER [74].

\section{Microscopy}

All images were obtained using a Leica SPE2 true scanning confocal microscope with $63 \times 1.4 \mathrm{NA}$ oil immersion objective. Images were recorded using Leica AF software. Calcofluorwhite (Sigma-Aldrich) staining was performed as described previously [68]. Calcofluor-white was used to allow cell segmentation for assays of cell morphology. For assays of cell viability, propidium iodide (Sigma-Aldrich) was added to the cells $(15 \mu \mathrm{g} / \mathrm{ml}$ final concentration) at the same point as the calcofluor-white. Staining was performed in a volume of $1 \mathrm{ml}$, allowed to proceed at room temperature for $10 \mathrm{~min}$, and the cells were washed twice in MM before visualisation. No cell fixation was applied. The vital dye FM4-64 (Molecular Probes) was used to visualise vacuolar morphology. The staining procedure has been described previously [12].

\section{Image analysis}

All microscopy image analysis was performed using ImageJ software. For analysis of cell morphology, cells were segmented using the BOA plugin of the quantitative imaging of membrane proteins (Quimp) package [75]. Cell elongation was then determined using the equation: $1-\left(\frac{4 \pi \text { area }}{\text { perimeter }^{2}}\right)$. Thirty cells were counted per dataset.

\section{Statistical analysis}

All statistical analysis was performed using Prism software, version $6.0 \mathrm{C}$ (Graphpad). In instances where only two samples were compared, significance was determined using an unpaired t test. In instances where multiple columns were compared, an ordinary one-way ANOVA was used with a Tukey's multiple-comparison post-test. All error bars represent mean \pm standard error of the mean (SEM). In all cases, the statistical test used, $p$-values and the number of samples analysed are highlighted in the figure legend for clarity.

\section{ACKNOWLEDGMENTS}

We would like to thank Dr. Elizabeth Henske, Dr. Ronit Weismann, Prof. Kentaro Nakano, Prof. Pilar Pérez, and Prof. Kaz Shiozaki for generously sharing resources. The research leading to these results (MB, SM) received funding from the European Community's Seventh Framework Programme (FP7/2007- 2013) under Grant Agreement N ${ }^{\circ} 281234$ (DEMCHILD), with additional funding from the Batten Disease Family Association, UK, and the Children's Batten Disease Foundation, USA. RB is funded by the University College London (UCL) MRC 4yr Doctoral Training Account in Life and Biomedical Sciences. Research in the Bähler laboratory $(C R, J B)$ is sup- 
ported by a BBSRC Research Grant [grant number BB/I012451/1] and a Wellcome Trust Senior Investigator Award [grant number 095598/Z/11/Z].

\section{SUPPLEMENTAL MATERIAL}

All supplemental data for this article are available online at www.microbialcell.com.

\section{CONFLICT OF INTEREST}

The authors declare no conflict of interest.

\section{COPYRIGHT}

(C) 2015 Bond et al. This is an open-access article released under the terms of the Creative Commons Attribution (CC BY) license, which allows the unrestricted use, distribution, and reproduction in any medium, provided the original author and source are acknowledged.

Please cite this article as: Michael E. Bond, Rachel Brown, Charalampos Rallis, Jürg Bähler and Sara E. Mole (2015). A central role for TOR signalling in a yeast model for juvenile CLN3 disease. Microbial Cell 2(12): 466-480. doi: 10.15698/mic2015.12.241

15. Codlin S, Haines RL, Mole SE (2008). btn1 affects endocytosis, polarisation of sterol-rich membrane domains and polarised growth in Schizosaccharomyces pombe. Traffic 9(6): 936-950.

16. Codlin S, Haines RL, Burden JJE, Mole SE (2008). btn1 affects cytokinesis and cell-wall deposition by independent mechanisms, one of which is linked to dysregulation of vacuole pH. J Cell Sci 121(17): 2860-2870.

17. Pears MR, Codlin S, Haines RL, White IJ, Mortishire-Smith RJ, Mole SE, Griffin JL (2010). Deletion of btn1, an orthologue of CLN3, increases glycolysis and perturbs amino acid metabolism in the fission yeast model of Batten disease. Molecular bioSystems 6(6): 10931102 .

18. Luiro K, Yliannala K, Ahtiainen L, Maunu H, Jarvela I, Kyttala A, Jalanko A (2004). Interconnections of CLN3, Hook1 and Rab proteins link Batten disease to defects in the endocytic pathway. Hum Mol Genet 13(23): 3017-3027.

19. Metcalfe DJ, Calvi AA, Seamann MNJ, Mitchison HM, Cutler DF (2008). Loss of the Batten disease gene CLN3 prevents exit from the TGN of the mannose 6-phosphate receptor. Traffic 11(11): 1905-1914.

20. Baryshnikova A, Costanzo M, Dixon S, Vizeacoumar FJ, Myers CL, Andrews $B$, Boone $C$ (2010). Synthetic genetic array (SGA) analysis in Saccharomyces cerevisiae and Schizosaccharomyces pombe. Methods Enzymol 470:145-179.

21. Willingham S, Outeiro TF, DeVit MJ, Lindquist SL, Muchowski PJ (2003). Yeast genes that enhance the toxicity of a mutant huntingtin fragment or alpha-synuclein. Science 302(5651): 1769-1772.

22. Ikai N, Nakazawa N, Hayashi T, Yanagida M (2011). The reverse, but coordinated, roles of Tor2 (TORC1) and Tor1 (TORC2) kinases for growth, cell cycle and separase-mediated mitosis in Schizosaccharomyces pombe. Biol Open 1(3): 110007.

23. Weisman R, Roitburg I, Schonbrun M, Harari R, Kupiec M (2007). Opposite effects of tor1 and tor2 on nitrogen starvation responses in fission yeast. Genetics 175(3): 1153-1162.

24. Matsuo T, Otsubo Y, Urano J, Tamanoi F, Yamamoto M (2007). Loss of the TOR kinase Tor2 mimics nitrogen starvation and activates the sexual development pathway in fission yeast. Molecular and cellular biology $27(8)$ : 3154-3164.

25. Cohen A, Kupiec M, Weisman R (2014). Glucose activates TORC2Gad8 protein via positive regulation of the $C A M P / C A M P-d e p e n d e n t$ protein kinase $A(P K A)$ pathway and negative regulation of the Pmk1 protein-mitogen-activated protein kinase pathway. J Biol Chem 289(31): 21727-21737. 
26. Ikeda K, Morigasaki S, Tatebe H, Tamanoi F, Shiozaki K (2008). Fission yeast TOR complex 2 activates the AGC-family Gad8 kinase essential for stress resistance and cell cycle control. Cell cycle 7(3): 358-364.

27. Hanyu Y, Imai KK, Kawasaki Y, Nakamura T, Nakaseko Y, Nagao K, Kokubu A, Ebe M, Fujisawa A, Hayashi T, Obuse C, Yanagida M (2009). Schizosaccharomyces pombe cell division cycle under limited glucose requires Ssp1 kinase, the putative CaMKK, and Sds23, a PP2A-related phosphatase inhibitor. Genes Cells 14(5): 539-554.

28. Levin DE (2005). Cell wall integrity signaling in Saccharomyces cerevisiae. Microbiol Mol Biol Rev 69(2): 262-291.

29. Matsuo T, Kubo Y, Watanabe Y, Yamamoto M (2003). Schizosaccharomyces pombe AGC family kinase Gad8p forms a conserved signaling module with TOR and PDK1-like kinases. EMBO 22(12): 3073-3083.

30. Madrid M, Fernandez-Zapata J, Sanchez-Mir L, Soto T, Franco A, Vicente-Soler J, Gacto M, Cansado J (2013). Role of the fission yeast cell integrity MAPK pathway in response to glucose limitation. BMC Microbiol 13:34.

31. Sugiura R, Toda T, Shuntoh H, Yanagida M, Kuno T (1998). pmp1+, a suppressor of calcineurin deficiency, encodes a novel MAP kinase phosphatase in fission yeast. EMBO J 17(1): 140-148.

32. Mutoh T, Nakano K, Mabuchi I (2005). Rho1-GEFs Rgf1 and Rgf2 are involved in formation of cell wall and septum, while Rgf3 is involved in cytokinesis in fission yeast. Genes Cells 10(12): 1189-1202.

33. Hartmuth S, Petersen J (2009). Fission yeast Tor1 functions as part of TORC1 to control mitotic entry through the stress MAPK pathway following nutrient stress. J Cell Sci 122(Pt 11): 1737-1746.

34. van Slegtenhorst M, Carr E, Stoyanova R, Kruger WD, Henske EP (2004). Tsc1+ and tsc2+ regulate arginine uptake and metabolism in Schizosaccharomyces pombe. J Biol Chem 279(13): 12706-12713.

35. Rallis C, Codlin S, Bahler J (2013). TORC1 signaling inhibition by rapamycin and caffeine affect lifespan, global gene expression, and cell proliferation of fission yeast. Aging Cell 12(4): 563-573.

36. Nakashima A, Sato T, Tamanoi $F$ (2010). Fission yeast TORC1 regulates phosphorylation of ribosomal $\mathrm{S} 6$ proteins in response to nutrients and its activity is inhibited by rapamycin. J Cell Sci 123(5): 777-786.

37. Nguyen AN, Shiozaki K (1999). Heat-shock-induced activation of stress MAP kinase is regulated by threonine- and tyrosine-specific phosphatases. Genes \& development 13(13): 1653-1663.

38. Mukaiyama $\mathrm{H}$, Nakase $\mathrm{M}$, Nakamura $\mathrm{T}$, Kakinuma $\mathrm{Y}$, Takegawa $\mathrm{K}$ (2010). Autophagy in the fission yeast Schizosaccharomyces pombe. FEBS Lett 584(7): 1327-1334.

39. Chandrachud U, Walker MW, Simas AM, Heetveld S, Petcherski A, Klein M, Oh H, Wolf P, Zhao WN, Norton S, Haggarty SJ, Lloyd-Evans E, Cotman SL (2015). Unbiased Cell-based Screening in a Neuronal Cell Model of Batten Disease Highlights an Interaction between $\mathrm{Ca} 2+$ Homeostasis, Autophagy, and CLN3 Protein Function. J Biol Chem 290(23): 14361-14380.

40. Toone WM, Kuge S, Samuels M, Morgan BA, Toda T, Jones N (1998). Regulation of the fission yeast transcription factor Pap1 by oxidative stress: requirement for the nuclear export factor $\mathrm{Crm} 1$ (Exportin) and the stress-activated MAP kinase Sty1/Spc1. Genes \& development 12(10): 1453-1463.

41. Halova L, Du W, Kirkham S, Smith DL, Petersen J (2013). Phosphorylation of the TOR ATP binding domain by AGC kinase constitutes a novel mode of TOR inhibition. J Cell Biol 203(4): 595-604.
42. Schmidt A, Bickle M, Beck T, Hall MN (1997). The yeast phosphatidylinositol kinase homolog TOR2 activates RHO1 and RHO2 via the exchange factor ROM2. Cell 88(4): 531-542.

43. Madrid $M$, Jiménez $R$, Sánchez-Mir L, Soto $T$, Franco A, VicenteSoler J, Gacto M, Pérez P, Cansado J (2015). Multiple layers of regulation influence cell integrity control by the PKC ortholog Pck2 in fission yeast. J Cell Sci 128(2): 266-80.

44. Sanchez-Mir L, Franco A, Martin-Garcia R, Madrid M, Vicente-Soler J, Soto T, Gacto M, Perez P, Cansado J (2014). Rho2 palmitoylation is required for plasma membrane localization and proper signaling to the fission yeast cell integrity mitogen- activated protein kinase pathway. Mol Cell Biol 34(14): 2745-2759.

45. Sanchez-Mir L, Soto T, Franco A, Madrid M, Viana RA, Vicente J, Gacto M, Perez P, Cansado J (2014). Rho1 GTPase and PKC ortholog Pck1 are upstream activators of the cell integrity MAPK pathway in fission yeast. PLoS One 9(1): e88020.

46. Nakano K, Arai R, Mabuchi I (2005). Small GTPase Rho5 is a functional homologue of Rho1, which controls cell shape and septation in fission yeast. FEBS Lett 579(23): 5181-5186.

47. Doi A, Kita A, Kanda Y, Uno T, Asami K, Satoh R, Nakano K, Sugiura $R$ (2015). Geranylgeranyltransferase Cwg2-Rho4/Rho5 module is implicated in the Pmk1 MAP kinase-mediated cell wall integrity pathway in fission yeast. Genes Cells 20(4): 310-323.

48. Viana RA, Pinar M, Soto T, Coll PM, Cansado J, Perez P (2013). Negative functional interaction between cell integrity MAPK pathway and Rho1 GTPase in fission yeast. Genetics 195(2): 421-432.

49. Garcia P, Tajadura V, Sanchez Y (2009). The Rho1p exchange factor Rgf1p signals upstream from the Pmk1 mitogen-activated protein kinase pathway in fission yeast. Mol Biol Cell 20(2): 721-731.

50. Wang S, Xu B, Liou LC, Ren Q, Huang S, Luo Y, Zhang Z, Witt SN (2012). alpha-Synuclein disrupts stress signaling by inhibiting polo-like kinase Cdc5/PIk2. Proc Natl Acad Sci USA 109(40): 16119-16124.

51. Tuxworth RI, Vivancos V, O'Hare MB, Tear G (2009). Interactions between the juvenile Batten disease gene, CLN3, and the Notch and JNK signalling pathways. Hum Mol Genet 18(4): 667-678.

52. Chan D, Citro A, Cordy JM, Shen GC, Wolozin B (2011). Rac1 protein rescues neurite retraction caused by G2019S leucine-rich repeat kinase 2 (LRRK2). J Biol Chem 286(18): 16140-16149.

53. Namekata K, Harada C, Taya C, Guo X, Kimura H, Parada LF, Harada $T$ (2010). Dock3 induces axonal outgrowth by stimulating membrane recruitment of the WAVE complex. Proc Natl Acad Sci U S A 107(16): 7586-7591.

54. Mocholi E, Ballester-Lurbe B, Arque G, Poch E, Peris B, Guerri C, Dierssen M, Guasch RM, Terrado J, Perez-Roger I (2011). RhoE deficiency produces postnatal lethality, profound motor deficits and neurodevelopmental delay in mice. PLoS One 6(4): e19236.

55. Bove J, Martinez-Vicente M, Vila M (2011). Fighting neurodegeneration with rapamycin: mechanistic insights. Nat Rev Neurosci 12(8): 437-452.

56. Sarkar S, Ravikumar B, Floto RA, Rubinsztein DC (2009). Rapamycin and mTOR-independent autophagy inducers ameliorate toxicity of polyglutamine-expanded huntingtin and related proteinopathies. Cell death and differentiation 16(1): 46-56.

57. Malagelada C, Jin ZH, Jackson-Lewis V, Przedborski S, Greene LA (2010). Rapamycin protects against neuron death in in vitro and in vivo models of Parkinson's disease. J Neurosci 30(3): 1166-1175.

58. Menzies FM, Huebener J, Renna M, Bonin M, Riess O, Rubinsztein DC (2010). Autophagy induction reduces mutant ataxin-3 levels and toxicity in a mouse model of spinocerebellar ataxia type 3 . Brain 133(Pt 1): 93-104. 
59. Zhao C, Yasumura D, Li X, Matthes M, Lloyd M, Nielsen G, Ahern K, Snyder M, Bok D, Dunaief JL, LaVail MM, Vollrath D (2011). mTORmediated dedifferentiation of the retinal pigment epithelium initiates photoreceptor degeneration in mice. J Clin Invest 121(1): 369-383.

60. Caccamo A, Maldonado MA, Majumder S, Medina DX, Holbein W, Magri A, Oddo S (2011). Naturally secreted amyloid-beta increases mammalian target of rapamycin (mTOR) activity via a PRAS40mediated mechanism. J Biol Chem 286(11): 8924-8932.

61. Dehay B, Bove J, Rodriguez-Muela N, Perier C, Recasens A, Boya P, Vila M (2010). Pathogenic lysosomal depletion in Parkinson's disease. J Neurosci 30(37): 12535-12544.

62. Zhou J, Tan SH, Nicolas V, Bauvy C, Yang ND, Zhang J, Xue Y, Codogno $P$, Shen HM (2013). Activation of lysosomal function in the course of autophagy via mTORC1 suppression and autophagosomelysosome fusion. Cell Res 23(4): 508-523.

63. Ries V, Henchcliffe C, Kareva T, Rzhetskaya M, Bland R, During MJ, Kholodilov N, Burke RE (2006). Oncoprotein Akt/PKB induces trophic effects in murine models of Parkinson's disease. Proc Natl Acad Sci U S A 103(49): 18757-18762.

64. Ling D, Song HJ, Garza D, Neufeld TP, Salvaterra PM (2009). Abeta42-induced neurodegeneration via an age-dependent autophagic-lysosomal injury in Drosophila. PLoS One 4(1): e4201.

65. Tuxworth RI, Chen H, Vivancos V, Carvajal N, Huang X, Tear G (2011). The Batten disease gene CLN3 is required for the response to oxidative stress. Hum Mol Genet 20(10): 2037-2047.

66. Bahler J, Wu JQ, Longtine MS, Shah NG, McKenzie A, 3rd, Steever $A B$, Wach A, Philippsen P, Pringle JR (1998). Heterologous modules for efficient and versatile PCR-based gene targeting in Schizosaccharomyces pombe. Yeast 14(10): 943-951.

67. Sato M, Dhut S, Toda T (2005). New drug-resistant cassettes for gene disruption and epitope tagging in Schizosaccharomyces pombe. Yeast 22(7): 583-591.
68. Moreno S, Klar A, Nurse P (1991). Molecular genetic analysis of fission yeast Schizosaccharomyces pombe. Methods Enzymol 194:795823.

69. Rorbach J, Richter R, Wessels HJ, Wydro M, Pekalski M, Farhoud M, Kuhl I, Gaisne M, Bonnefoy N, Smeitink JA, Lightowlers RN, Chrzanowska-Lightowlers ZM (2008). The human mitochondrial ribosome recycling factor is essential for cell viability. Nucleic Acids Res 36(18): 5787-5799.

70. Schonbrun M, Laor D, Lopez-Maury L, Bahler J, Kupiec M Weisman $R$ (2009). TOR complex 2 controls gene silencing, telomere length maintenance, and survival under DNA-damaging conditions. Mol Cell Biol 29(16): 4584-4594.

71. Tatebe H, Shiozaki K (2003). Identification of Cdc37 as a novel regulator of the stress-responsive mitogen-activated protein kinase. Mol Cell Biol 23(15): 5132-5142.

72. Rallis C, Lopez-Maury L, Georgescu T, Pancaldi V, Bahler J (2014). Systematic screen for mutants resistant to TORC1 inhibition in fission yeast reveals genes involved in cellular ageing and growth. Biol Open 3(2): 161-171.

73. Wagih O, Parts L (2014). gitter: a robust and accurate method for quantification of colony sizes from plate images. G3 (Bethesda) 4(3): 547-552.

74. Mi H, Muruganujan A, Thomas PD (2013). PANTHER in 2013: modeling the evolution of gene function, and other gene attributes, in the context of phylogenetic trees. Nucleic Acids Res 41(Database issue): D377-386.

75. Bosgraaf L, van Haastert PJ, Bretschneider T (2009). Analysis of cell movement by simultaneous quantification of local membrane displacement and fluorescent intensities using Quimp2. Cell Motil Cytoskeleton 66(3): 156-165.

76. Chen D, Toone WM, Mata J, Lyne R, Burns G, Kivinen K, Brazma A Jones N, Bahler J (2003). Global transcriptional responses of fission yeast to environmental stress. Mol Biol Cell 14(1): 214-229. 